\title{
A Multi-model Kalman Filter Clock Synchronization Algorithm based on Hypothesis Testing in Wireless Sensor Networks
}

\author{
Xiali Li ${ }^{1, a}$, Shaona $\mathrm{Yu}^{1}$, Yuan $\mathrm{Lin}^{1}$, Min Xi ${ }^{2}$ \\ ${ }^{1}$ Computer Science and Technology, Minzu University of China, Beijing, China, \\ ${ }^{2}$ Computer Science and Technology, Xi'an JiaoTong University,Shannxi, China \\ axiaer_li@163.com
}

Keywords: WSN;Multi-model;Kalman Filter;Hypothesis Testing;Clock Offset

\begin{abstract}
Accurate and efficient clock synchronization algorithm is very important in Wireless Sensor Networks (WSN). Considering the physical nature of sensors, a multi-model kalman filter clock synchronization algorithm based on experiments and hypothesis testing theory is proposed. The sensor experiments on the base of TelosB platform demonstrate that sensor clock system switches between different models. Based on this observation, a general multi-model kalman filter to describe clock offset is presented. Hypothesis testing method is used to switch the model between the first-order kalman filter and second-order kalman filter. Experiments show that the proposed algorithm can trace clock offset effectively.
\end{abstract}

\section{Introduction}

Clock synchronization is important in wireless sensor network (WSN). Sorting logs for systems diagnostics, coordinating of scheduling events in some media access control(MAC) protocols and providing accurate timestamps for cryptographic protocols all need precise clock synchronization mechanism.

Ref. [1] and [2] think that limited power of sensor nodes in WSN make it impossible to achieve clock synchronization by unlimited passing information among sensor nodes. Due to limited power, phase noise, thermal noise and degradation rate, the sensor clock crystal frequency is not very precise. Ref. [3] think that sensors are usually deployed in a complex environment in which temperature, humidity and other factors may affect the work of the clock. The features of WSN make it very difficult to use the existing methods to realize clock synchronization.

Most clock synchronization protocols such as those presented in [4, 5, 6] use Stochastic Differential Equations (SDEs) to describe the clock model on the base of oscillator physical nature. Protocols presented in $[7,8]$ use a constant model to describe the relatively stable clock system with "white noise" which reflects phase noise and other random factors. Researchers usually build clock skew modeling by using the first-order autoregressive model. Ref. [4] uses second-order kalman filter model to describe clock skew. However, most of the models are only suitable for special environments and are not general. In order to alleviate the various difficulties of clock synchronization, this paper proposes a general clock synchronization algorithm based on the multi-model kalman filter and hypothesis testing theory. From the sensor experiments on the base of TelosB platform, it is observed that sensor clock system switch between different models. Based on this analysis, a general multi-model kalman filter model is put forward for describing clock oscillator drift. Expectation-maximum(EM) algorithm is used to estimate parameters in the model and hypothesis testing method is used to realize model switch between the first-order kalman filter and second-order kalman filter. Finally, the performance gains of our algorithm is demonstrated by using different kalman filter models based on experiment data.

\section{Sensor Clock Experiment and Analysis}


Sensor Clock Experiment Set.In order to study clock model from the perspective of crystal oscillator, the following experiments are implemented. Clock skew test of 10 wireless sensor nodes was conducted. Node model is TPR2400CA, the platform is TelosB, CPU frequency is from $2400 \mathrm{MHz}$ to $2483.5 \mathrm{MHz}$. It is noteworthy that TPR2400CA is the mainstream hardware for wireless sensor networks. The test environment was the enclosed area with air conditioning where temperature was controlled to be $25 \pm 5^{\circ} \mathrm{C}$. All 10 sensors were deployed at different locations to monitor temperature and send temperature messages to the receiver at 500s intervals. Receiver was connected to the computer with a stable electricity supply. When temperature message was received, the time when the message was sent exactly will be recorded. This recorded time is the basis of time synchronization test. All sensor nodes were powered by the same type of battery.

The experiment was repeated 10 times. All the sensor nodes have the same time offset rule was observed. Here the paper randomly selected three nodes data to explain the experiment results.

Results and Analysis.Before node power is depleted, each node can upload about 670 data to the receiver. During the last period when battery is nearly depleted, the received data is very unstable. So only the first 600 messages were used to analyze the data. Fig. 1 shows the clock offset of three nodes at different time. The dotted line shows the ideal clock, the other three curves show the three clock offset of the sensor nodes. From Fig.1, it can be seen that every clock offset curve has a deviation with the ideal curve and the deviation has a gradual upward trend. The first 300 sampling points are relatively stable, while the remaining nodes deviate from the ideal curve rapidly. All the information shows that random noise is not the only factor that affects clock offset and these clocks are slower than the ideal clock. From Fig. 1, it can be observed that the clock skew has the model-switching phenomenon.

A single model can not effectively describe the sensor node clock changes from the observation and analysis of the experiments.

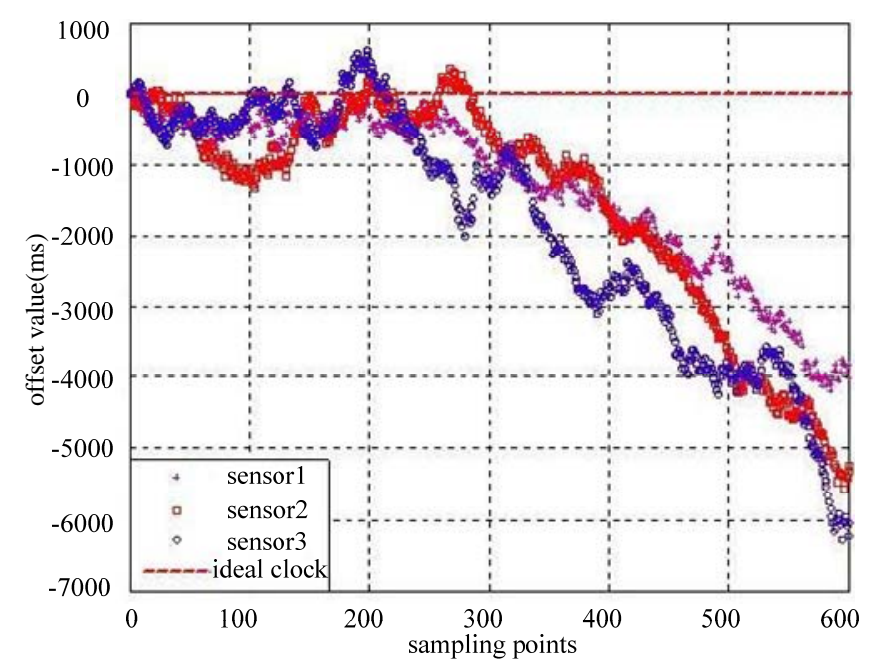

Figure1 the clock offset of three nodes

\section{Multi-model Kalman Filter Clock Model}

Clock model of the wireless sensor node is very complex. Therefore, establish a reliable clock model to realize clock synchronization is important. As the clock oscillator frequency is a key factor that affects clock, crystal oscillator characteristics are the focus in this section.

From Ref.[10], the following general clock offset model is gotten,

$$
o[n]=o[n-1]+s[n] \tau[n]+0.5 \gamma[n] \tau^{2}[n]+\vartheta[n]
$$

where $o[n]$ is clock offset, $s[n]$ is the clock skew at sampling time $n, \tau[n]$ is sampling time interval, $\vartheta[n]$ is the combination of offset noise, skew noise and aging rate noise, and $\gamma[n]$ is the aging rate. 
As in [11], $x[n]$ represents the n-th value of the clock variable, $y[n]$ represents the n-th clock observation, $F$ represents the state transition matrix, $H$ represents the observation matrix, $\mu[n]$ and $\zeta[n]$ represents the white noise covariance matrix $Q$ and $R$ respectively, the following Kalman Filter can be obtained.

$$
\begin{aligned}
& x[n]=F x[n-1]+\mu[n] \\
& y[n]=H x[n]+\zeta[n]
\end{aligned}
$$

Two kinds of kalman filter models according to the above definitions are discussed here.

Case 1: If the aging rate is zero, the clock model is regarded as a constant velocity model. The following set for the kalman filter is given

$$
x=[o[n] \quad s[n]]^{T}, F=\left[\begin{array}{ll}
1 & \tau \\
0 & 1
\end{array}\right], H=\left[\begin{array}{ll}
1 & 0
\end{array}\right], \mu[n]=[\vartheta[n] \quad \eta[n]]^{T} \text { where } \mu[n] \text { is state noise and }
$$

$\zeta[n]$ is observation noise. Thus the model is the first-order kalman filter of the clock offset.

Case 2: If the aging rate is a non zero constant, the clock model is regarded as an accelerated motion model. The aging rate is a small perturbation around the mean random process. Assuming a tiny disturbance for a white noise, set the kalman filter to be the following

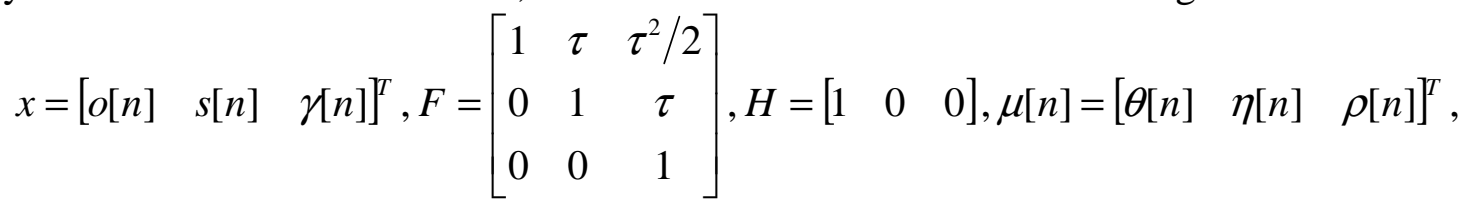

where $\rho[n]$ is aging rate noise, $\zeta[n]$ is observation noise. Then the model becomes the second-order kalman filter of the clock offset.

Ref. [10] has used first-order kalman filter to track the clock synchronization and the results shown that first-order kalman filter can only trace the observation data of some sampling points. Ref. [12] has used second-order kalman filter to track the clock synchronization and the results shown that second-order kalman filter has proper performance in tracing the latter sampling points.

Ref. [10] and Ref. [12] show that whether first-order kalman filter or second-order Kalman filter clock model can not trace clock offset very well independently. Further analysis demonstrates that the clock offset can be divided into two parts. The first part approximates to meet the first-order kalman filter model and the other part approximates to satisfy the second-order kalman filter model. In this paper, a multi-model kalman filter clock synchronization model is proposed. Using this method, the dynamic system can switch between first-order kalman filter and second-order kalman filter.

\section{Hypothesis Testing for Multi-model Kalman Filter}

Hypothesis Testing.The covariance $S_{t}$ at time $t$ in the iterative process of the kalman filter can be obtained by the following equation

$$
S_{t}=H_{t} V_{t, t-1} H^{T}+R_{t}
$$

where $H_{t}$ represents observation matrix model at time $t$ and is used to map the actual state space to the observation space, $R_{t}$ is white noise covariance matrix value at time $t, V_{t, t-1}$ is the error covariance matrix for estimation value based on observational data of the $\mathrm{t}-1$ moment prior to the time t, $H$ represents the initial set and has different value for first-order filter and second-order filter.

For the system with two models, the transfer of the model can be reflected in the changes of the error covariance. That is, if the model is transferred, significant changes in error covariance will occur. Therefore, hypothesis testing is used to determine the transfer of this model. Based on hypothesis testing theory [11], the following null hypothesis and alternative hypothesis can be gotten,

$H_{0}: S_{t}=S_{t-1}, H_{1}: S_{t} \neq S_{t-1}$

where $H_{0}$ is rejection region and $H_{1}$ is acceptance region. 
Using (3) and (4), Eq. (5) can be obtained,

$P\left\{\right.$ reject $H_{0} \mid H_{0}$ istrue $\}=P\left\{\left(D_{t} \leq K_{1}\right) \cup\left(D_{t} \geq K_{2}\right)\right\}=s_{h}$

where $s_{h}$ is the significance level, $K_{1}$ and $K_{2}$ are threshold, $K_{1}<K_{2}, D_{t}$ is rejection region.

Based on Eq. (4) and Eq. (5), the following rejection region $D_{t}$ will be gotten,

$D_{t} \leq K_{1}=\chi_{1-s_{h} / 2}^{2}(m)$ or $D_{t} \geq K_{2}=\chi_{1-s_{h} / 2}^{2}(m)$

where $m$ is degrees of freedom. Using the method of hypothesis testing, not only computing the transition probability matrix is avoided, but also judging when the state of the kalman iterative process transfer is avoided.

Clock Offset Tracing Using Multi-model Algorithm.The only observational data is clock skew in observing system. The observation error $U_{t}$ is one-dimensional vector. Ref. [11] indicates that hypothesis testing is usually $5 \%, 1 \%$ or $0.1 \%$ significance level.

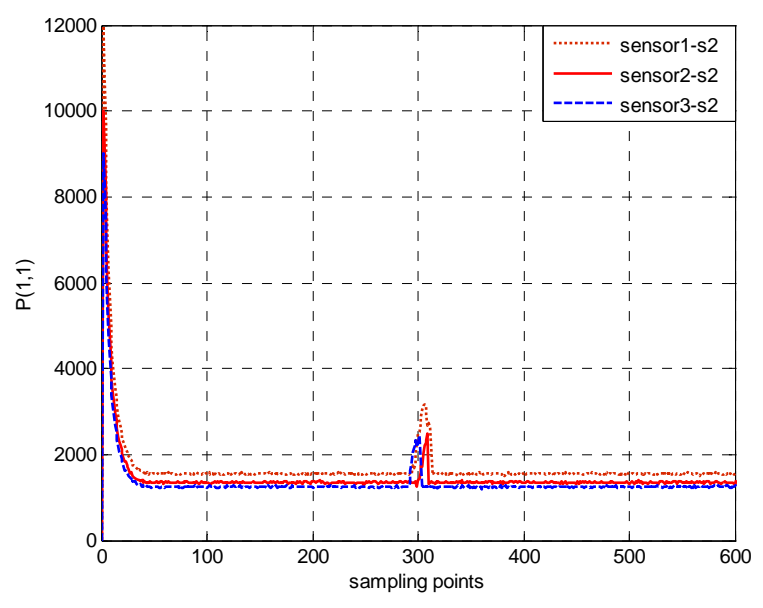

Figure 2 the first item of error covariance matrix
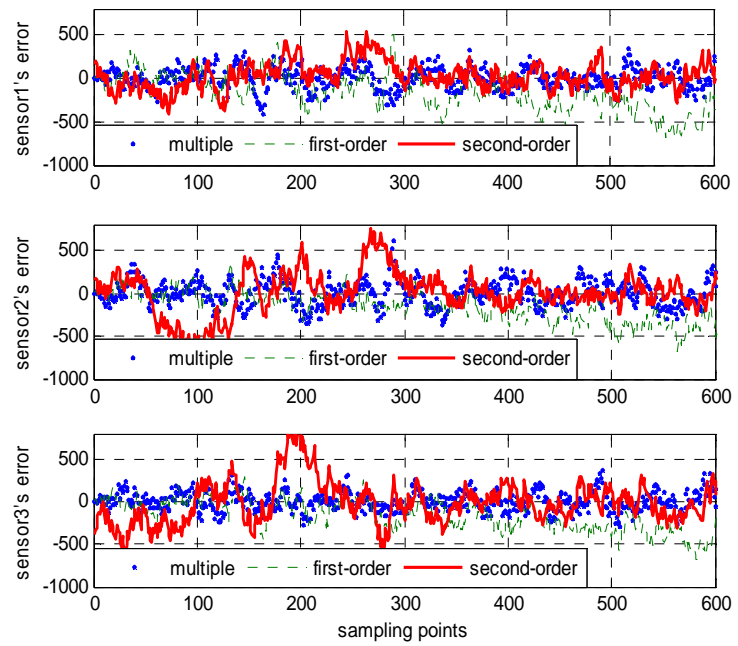

Figure 3 Error comparison result between different models

If the hypothesis testing probability is less than $s_{h}$, it will reject the null hypothesis. The more significantly lower than $s_{h}$, it will be the more inclined to accept the null hypothesis. But at the same time, it will increase the risk of error null hypothesis (Type II error described in [11]). Therefore, it does not have statistical nature. As how to choose significant level is involved in balance significance and effectiveness of hypothesis testing, the significant level is generally in the probability interval from Type I to Type II error probability. Here 1\% is used to be as the significance level to verify the multi-model kalman filter performance.

Fig. 2 shows the tracking of the first item $(\mathrm{P}(1,1))$ of error covariance matrix of multimode clock offset tracking. $(\mathrm{P}(1,1))$ converges to a fixed value in a very short period of time. There is a small fluctuation at approximately the 300th sampling point and it demonstrates that a model switch occurs at this time.

Fig. 3 shows that the error comparison between observation value and estimated values of different models. From Fig.3, it can be seen that the error in the last part of the first order filter and the first part of second-order filter are greater than those in multi-model. Fig. 2 and Fig. 3 demonstrate that the multi-model kalman filter has a higher accuracy compared with first-order filter and second-order filter.

\section{Conclusion}

The multi-model kalman filter method in clock offset tracking has smaller error compared with the simple first-order or second-order methods. The multi-model algorithm is divided into learning phase and tracking phase. It continuously collects clock synchronization messages of the clock model in 
learning stage. The messages are the basis for tracking in the next stage. In the tracking phase, the algorithm synchronizes the clock of the wireless sensor nodes using the multi-model kalman filter. The computing function of the two stages is configured in the server, so the algorithm does not have too much impact on the energy consumption of sensor nodes. Therefore, the multi-model kalman filter algorithm based on hypothesis testing has good performance than first-order and second- order filter.

\section{Acknowledgment}

This paper is supported by the National Key Technologies R\&D Program of China(No.2009BAH41B07) and the Real-time POCS data reconstruction based on conjugate gradient and Graphic Processing Unit project(N0.1112KYQN38).

\section{References}

[1] Hamilton BR, Ma X, Zhao Q, et al. ACES: adaptive clock estimation and synchronization using Kalman filtering[C]: ACM, 2008: 152-162.

[2] Sullivan D, Allan D, Howe D, et al. Characterization of clocks and oscillators. National Inst. of Standards and Technology, Boulder, CO. Time and Frequency Div, 1990.

[3] Sundararaman B, Buy U, Kshemkalyani AD. Clock synchronization for wireless sensor networks: a survey[J]. Ad Hoc Networks, 2005, 3 (3): 281-323.

[4] Galleani L, Sacerdote L, Tavella P, et al. A mathematical model for the atomic clock error[J]. Metrologia, 2003, 40: S257-S264.

[5] Kim KS, Lee BG. Kalp: A kalman filter-based adaptive clock method with low-pass prefiltering for packet networks use[J]. IEEE Transactions on Communications, 2000, 48 (7): 1217-1225.

[6] Auler LF, d'Amore R. Adaptive Kalman Filter for Time Synchronization over Packet-Switched Networks: An Heuristic Approach[C], 2007: 1-7.

[7] Veitch D, Babu S, Pàsztor A. Robust synchronization of software clocks across the internet[C]: ACM New York, NY, USA, 2004: 219-232.

[8] Allan DW. Time and frequency(time-domain) characterization, estimation, and prediction of precision clocks and oscillators[J]. IEEE transactions on ultrasonics, ferroelectrics, and frequency control, 1987, 34 (6): 647-654.

[9] Elson JE. Time synchronization in wireless sensor networks[D]: University of California Los Angeles, 2003.

[10] Xiali Li,et al. A General Clock Synchronization Method Based on Kalman Filter Model in Wireless Sensor Networks[C], International Conference on Consumer Electronics, Communications and Networks , 2012.

[11] Yu Yang, Zhulin An, Yongjun Xu, Xiaowei Li, Canfeng Che. Passive Loss Inference in Wireless Sensor Networks Using EM Algorithm[J], Wireless Sensor Network ,2010,2(7):512-519.

[12] Xiali Li,et al. Clock Synchronization Using Expectation-Maximization Algorithm in Wireless Sensor Network[C],international Conference on Computer Science and Service System,2012:in press. 